\title{
Analyse Multidimensionnelle Des Facteurs D’intégration Des Tice: Etude De Cas À Travers Le Programme Genie Marocain
}

\author{
Samiha Benfaress \\ Formation Doctorale en Didactique des Sciences et Ingénierie Pédagogique, \\ Centre d'Etudes Doctorales «Sciences et Techniques », \\ Faculté des Sciences Dhar El Mahraz, Fès, Maroc \\ Moncef Zaki \\ Anouar Alami \\ Laboratoire Interdisciplinaire de Recherche en Didactique \\ des Sciences et Techniques (LIRDIST), \\ Université Sidi Mohammed Ben Abdellah, Fès, Maroc
}

doi: 10.19044/esj.2016.v12n19p164 URL:http://dx.doi.org/10.19044/esj.2016.v12n19p164

\begin{abstract}
This article focuses on the integration of ICT in high-school level within the framework of teaching of sciences of Life and Earth. The experimentation was conducted with a sample of 112 Moroccan teachers of the region of Fez - Boulemane, who responded to a questionnaire of 16 modalities relating to the availability and the use of ICT tools, educational management of ICT in classroom, and the trainings and competencies in ICT and Informatics of the teachers. In order to make an original contribution to the study of the integration of ICT, we adopted a multidimensional analysis in the treatment of the answers of the teachers. Thus, thanks to a Factorial Analysis of Multiple Correspondence (FAMC), we could take account of the links which exist between the various modalities of our questionnaire to get to identify the most discriminating factors in the integration of ICT, like their order of importance. The results of this analysis led to three factorial axes cumulating an absolute inertia of $70 \%$, respectively representing «the pedagogical contribution and the fundamental conditions of a success of the integration of ICT», «the advanced competencies in ICT and Informatics» and «the difficulties of use of ICT in classroom». These axes, in this hierarchy, represent finally the most discriminating factors for a successful integration of ICT.
\end{abstract}

Keywords: Integration of ICT, Secondary education, Sciences of Life and Earth, Multidimensional Analysis, Discriminating factors 


\section{Résume}

Cet article s'intéresse à l'intégration des TICE au niveau du secondaire qualifiant dans le cadre de l'enseignement des Sciences de la Vie et de la Terre. L'expérimentation a été effectuée auprès d'un échantillon de 112 enseignants marocains de la région de Fès - Boulemane, qui ont répondu à un questionnaire de 16 modalités portant sur la disponibilité et l'utilisation des outils TICE, la gestion pédagogique des TICE en classe, et les formations et compétences en TICE et Informatique des enseignants. Afin d'apporter une contribution originale à l'étude de l'intégration des TICE, nous avons adoptée une analyse multidimensionnelle dans le traitement des réponses des enseignants. Ainsi, grâce à une Analyse Factorielle de Correspondance Multiple (AFCM), nous avons pu tenir compte des liaisons qui existent entre les diverses modalités de notre questionnaire, pour arriver à dégager les facteurs les plus discriminants dans l'intégration des TICE, ainsi que leur ordre d'importance. Les résultats de cette analyse ont conduit à trois axes factoriels cumulant une inertie absolue de $70 \%$, représentant respectivement « l'apport pédagogique et les conditions fondamentales d'une réussite de l'intégration des TICE », " les compétences avancées en TIC et Informatique » et " les difficultés d'utilisation des TICE en classe ». Ces axes, dans cette hiérarchie, représentent finalement les facteurs les plus discriminants pour une intégration réussie des TICE.

Mots clés: Intégration des TICE, Enseignement Secondaire, Sciences de la Vie et de la Terre, Analyse Multidimensionnelle, Facteurs discriminants

\section{Introduction}

L'utilisation des Technologies de l'Information et de la Communication (TIC) constitue aujourd'hui une pratique sociale qui se développe à très grande vitesse, en intégrant tous les secteurs d'activités, notamment celui de l'éducation. L'augmentation considérable d'informations et le développement extraordinaire de connaissances font que l'intégration des TIC dans l'enseignement et l'apprentissage soit devenue aujourd'hui un enjeu incontournable dans le monde éducatif de toute société (Matoussi, 2006). Diverses études et recherches sont donc menées à propos de l'intégration des TIC dans la sphère de l'éducation ; et les résultats de ces travaux ne sont pas toujours concordants (AFD, 2010 ; Alj, O. et Benjelloun, N., 2016). Une des raisons majeures à ces divergences, et que l'on pourrait avancer en toute rigueur, semble être les caractéristiques intrinsèques à la noosphère du système éducatif lui-même, ainsi que les spécificités du système d'enseignement faisant l'objet d'étude (Eurydice, 2011). Ainsi, les politiques éducatives et les systèmes d'enseignement de chaque pays, et non 
seulement, vont avoir un effet non négligeable sur l’intégration des TICE, ainsi que sur sa réussite auprès des apprenants : c’est pourquoi nous avons cherché à apporter une nouvelle contribution à la problématique de l’intégration des TICE dans le système éducatif marocain.

Les Hautes Orientations du discours du Roi du Maroc (23 Avril 2001) sur le « Maroc dans la société globale de l'information et du savoir », ont conduit le Ministère de l'Éducation Nationale à mener depuis maintenant quelques années une politique en faveur de l'usage des Technologies de l'Information et de la Communication. Cette même politique est au demeurant celle qui est préconisée par La Charte Nationale de l'Education et de la Formation de 1999 (COSEF Maroc, 1999). Ainsi, plusieurs grands programmes nationaux ont été lancés ces dernières années pour l’intégration des TICE dans le système éducatif marocain :

- le programme GENIE 2006-2013,

- le plan d'urgence 2009-2012 (Espace 1, Projet 10),

- la Stratégie Maroc Numéric 2020,

- le programme INJAZ.

En outre, un portail national « Taalimtice ou portailtice » a été mis en place en 2011 dans l'objectif de permettre à tous les acteurs du système éducatif marocain de se former et de s'informer sur les TICE, d'échanger des informations, et de participer activement au développement et à la diffusion des TICE.

Nous avons donc cherché à contribuer à l'étude de l'état d'avancement de l'intégration des TICE au Maroc, en allant explorer la question du côté des enseignants des Sciences de la Vie et de la Terre (SVT) au niveau de l'enseignement secondaire. Nous avons voulu adopter une approche d'analyse de la situation différente de celles adoptées jusqu’à maintenant par les diverses études et recherches effectuées au Maroc (El Ouidadi, O., 2012 ; Lakdim, A et al. , 2012 ; Alj, O. 2014 ; Ouazzani Touhami, A., 2014 ; Ouazzani Touhami, A. and al, 2014 ; Droui, M., 2015) dans l'objectif de mieux cerner les questions relatives à l'efficacité des politiques menées en termes d'intégration des TICE, aux conditions de sa réussite, ou encore aux difficultés rencontrées par les enseignants lors de l’intégration des TIC en classe.

\section{Cadre théorique et problématique}

Les travaux de recherche durant cette dernière décennie sur l'intégration des TICE au niveau de l'enseignement secondaire marocain, constitue un cadre théorique assez riche, permettant d'introduire notre propre problématique de recherche.

Ces travaux se sont intéressés aux effets de l’intégration d’outils TIC dans la transition secondaire-supérieur, comme c'est le cas dans le travail de 
A. Ouazzani (2014) qui a travaillé sur les difficultés conceptuelles en optique géométrique et qui a démontré quelques effets de remédiassions auprès des étudiants par le biais de l'utilisation d'un Atelier Java d'Optique Géométrique (AJOG). Les recherches de M. Droui (2015) s’inscrivent aussi dans le même type de problématique ; il montre qu'un apprentissage actif basé sur la simulation et l'ExAO favorise une meilleure appréhension des lois de Newton.

Pour notre part, nous nous intéressons aussi à l'intégration des TICE, mais selon une approche qui reste complémentaire à celles des deux travaux de recherche précédents. Notre problématique est proche de celles de O. El Ouidadi (2012), Maouni (2014), El Madhi (2014) et O. Alj (2014) qui font respectivement un état des lieux sur l'intégration des TICE dans l'enseignement secondaire marocain, par le biais de l'interrogation des enseignants : les trois premiers se sont intéressés aux Sciences de la Vie et de la Terre (SVT), comme c'est le cas pour nous ; et le dernier aux sciences physiques et à l'algorithmique. Ces travaux, basés tous sur des analyses statistiques descriptives, s'accordent sur plusieurs contraintes relatives à l'intégration des TICE :

- Manque de compétences techno-pédagogique

- $\quad$ Manque de ressources numériques

- $\quad$ Disparité des équipements informatiques entre les établissements et difficulté de maintenance des équipements

- Disparité des compétences et de la formation des enseignants en TICE

Notre approche de la problématique de l'intégration des TICE diffère des précédentes, par sa méthodologie d'analyse des réponses des enseignants interrogés. En effet, dans notre problématique, nous chercherons grâce à des méthodes statistiques multifactorielles de répondre aux questions suivantes :

- Quelles sont les liaisons qui peuvent exister entre les contraintes d'intégration des TICE ?

- Quelles interprétations peut-on donner aux tendances que représentent ces liaisons?

- $\quad$ Quelles sont les quantifications, en termes de degré d'importance, de ces tendances ; et quelles sont donc celles qui sont prioritaires ?

- $\quad$ Et enfin, discerner entre les contraintes les plus discriminantes face à la question de l'intégration des TICE, de celles dont l'effet est sans grande importance pour cette intégration.

\section{Expérimentation et méthodologie de recherche}

Notre problématique porte sur l'intégration des TICE, selon une investigation qui cible les enseignants du secondaire de Sciences de la Vie et 
de la Terre (SVT). Nous avons donc cherché à traiter cette question de manière exhaustive dans la région de Fès-Boulemane, région rattachée à notre Université Sidi mohammed Ben Abdellah de Fès.

L’Académie Régionale de l'Education et de la Formation (AREF) de Fès-Boulemane (représentant institutionnel et régional du Ministère de l'Education et de la Formation) constitue au niveau national une des académies des plus importantes sur l'ensemble du territoire marocain, avec un total de 253 enseignants de SVT du secondaire qualifiant. Ainsi, après autorisation officielle de l'AREF de Fès-Boulemane, nous avons pu travailler avec un échantillon de 112 enseignants de SVT, se répartissant en 97 enseignants relevant de la zone urbaine (Fès, Missour et Séfrou) et 15 enseignants de la zone rurale (Ribat El Kheil, El Menzel, Outat El Haj, Immouzer Marmoucha et Guigou). Nous pouvons ainsi largement avancer pour notre recherche, l'hypothèse d'exhaustivité et de représentativité de notre échantillon pour la population d'enseignants de SVT de l'AREF de Fès-Boulemane.

L’approche expérimentale adoptée pour notre recherche, a été fondée sur l'interrogation de notre échantillon à l'aide d'un questionnaire, dont la passation a duré sept mois au cours de l’année académique 2013 /2014. Par ailleurs, la taille relativement importante de l'échantillon (112 enseignants), permet de conduire dans de bonnes conditions l'étude des facteurs discriminants de l'intégration des TICE à partir d'une analyse multidimensionnelle.

Le questionnaire (cf. Annexe A), composé de 16 items, a été établi autour des points suivants :

- Disponibilité et utilisation des outils TICE.

- Gestion pédagogique des TICE en classe.

- $\quad$ Formations et compétences en TICE et Informatique.

Ces trois points constituent les éléments fondamentaux pour traiter la problématique de l'intégration des TICE, on les retrouve d'ailleurs en partie dans les travaux de recherches antérieures, notamment chez El Ouidadi (2012) et El Madhi (2014) pour les SVT, et Alj (2014) et Droui (2015) pour la physique. Ces travaux antérieurs, tous basés sur des analyses de statistique descriptive, ont permis de relever plusieurs facteurs qui font obstacle à l'intégration des TICE, comme par exemples :

- l'insuffisance de formation des enseignants,

- $\quad$ le manque d'infrastructure et d'équipement dans les établissements,

- $\quad$ le manque de maintenance régulière des équipements.

- $\quad$ le sureffectif des élèves,

- l'absence de planification et de soutien technique pour intégrer les TICE, 
L'identification de tous ces facteurs est certes fondamentale et importante pour mener à bien la question d'intégration des TICE ; seulement, l'utilisation de simples statistiques descriptives ne peut pour autant nous renseigner sur des questions encore plus fondamentales :

- $\quad$ Quelles liaisons peuvent exister entre ces différents facteurs ?

- $\quad$ Quels sont les facteurs qui contribuent le plus à ces liaisons ?

- $\quad$ Quel est l'ordre d'importance des facteurs discriminants qui explique ces liaisons?

Pour répondre à toutes ces questions, Il s'agira bien sûr de mener une analyse descriptive, mais à caractère multidimensionnelle. L'Analyse Factorielle de Correspondance Mutiple (AFCM) semble être bien appropriée à ce type d'analyse, d'autant plus que nous disposons de 16 items à plusieurs modalités, autrement dit de 16 variables qualitatives, que nous traitons auprès d'un échantillon relativement important de 112 enseignants. Une telle analyse, basée sur le principe mathématique de réduction de dimension, va permettre de déterminer, en termes de liaisons, les modalités des variables qui sont les plus discriminantes, et d'en déduire la nature des facteurs composés sur la base des liaisons de ces modalités, au même titre que leur ordre d'importance.

\section{Interprétation des résultats de l'analyse factorielle de correspondance multiple (AFCM)}

Le traitement des réponses des 112 enseignants a été réalisé par une AFCM à l'aide du logiciel de traitement de données SPSS (Statistical Pakage for the Social Sciences) en version 21. En partant du principe fondamental de réduction de dimensions de l'AFCM, nous avons cherché dans un premier temps à identifier les modalités des variables dont les contributions relatives à la construction des axes factoriels sont très négligeables. Une première AFCM effectuée avec l'ensemble des 16 items a permis d'identifier ces variables, ce qui a permis d'éliminer les questions relatives aux items suivants :

- $\quad$ Disponibilité d'un ordinateur

- $\quad$ Temps de navigation sur Internet

- $\quad$ Présence d'une salle d'informatique dans l'établissement

- $\quad$ Utilisation de la simulation en cours

- $\quad$ Utilisation des TICE pour la planification des cours

Une seconde AFCM a été effectuée, dans laquelle nous n'avons rendu actif que les 11 items restants sur les 16 de départ. Par comparaison aux résultats de l'AFCM avec les 16 items de départ, la seconde AFCM a donné lieu quasiment à la même répartition de mesures de discrimination pour les 11 items restants : cela confirme encore une fois la justification 
d'élimination des questions relatives aux cinq items ci-dessus, et que leur élimination n'affecte en rien l'essentiel de l'information contenue dans les réponses de l'ensemble des enseignants au questionnaire.

La seconde AFCM, limitée à 11 items, a conduit à trois valeurs propres $\square_{1}=4,338, \square_{2}=2,176$ et $\square_{3}=1,085$, représentant toutes les trois une inertie expliquée valant 69,081\%. Ainsi, l’inertie expliquée par les trois premiers axes factoriels avoisine les $70 \%$, c'est pourquoi nous nous limiterons dans l'interprétation de l'AFCM aux trois premiers axes factoriels. Par ailleurs, du fait que ces trois valeurs propres soient chacune d'ordre de multiplicité $1\left(\square_{1}>\square_{2}>\square_{3}\right.$ ), chacun des trois axes factoriels sera interprété séparément.

\section{Interprétation du $\mathbf{1}^{\mathrm{er}}$ axe factoriel}

L'AFCM a conduit à une inertie absolue de 39,43\% pour le 1er axe factoriel, soit un peu plus du tiers de l'inertie totale du nuage représenté par l'ensemble des variables actives, ce qui représente une part importante de l'ensemble de l'information traitée. Les variables qui ont le plus contribué à la formation de ce premier axe factoriel sont les suivantes :

Tableau 1 : Variables à fortes contributions relatives dans la construction du $1^{\mathrm{er}}$ axe factoriel

\begin{tabular}{|c|c|}
\hline Intitulé des variables (Modalités) & $\begin{array}{c}\text { Contribution relative } \\
\text { à l'Axe } 1\end{array}$ \\
\hline Interactivité des élèves (E_Interact_Oui et E_Interact_Non) & 0,933 \\
\hline Utilisation des Outils TICE (O_Tice_F et O_Tce_NF) & 0,907 \\
\hline $\begin{array}{c}\text { Participation des élèves à l'usage des TICE (E_Tice_Oui et } \\
\text { E_Tice_Non) }\end{array}$ & 0,898 \\
\hline Compétence en Office (OF_S (Suffisante) et OF_INS (Insuffisante)) & 0,867 \\
\hline Type de formation (F_Institu (Institutionelle) et F_Auto & 0,448 \\
(Autoformation) & 0,260 \\
\hline Formation en TICE (F_Tice_Oui et F_Tice_Non) & \\
\hline
\end{tabular}

Dans le premier plan factoriel (Axe1, Axe2) (Voir Figure 1), on constate qu'il y a une parfaite opposition par rapport au $1^{\mathrm{er}}$ axe factoriel entre les modalités représentant la présence (Oui) et l'absence (Non) de ces variables. Ainsi, le premier axe factoriel sépare, d'une part (du côté des composantes négatives) les modalités E_Interact_Oui , O_Tice_F , E_Tice_Oui, OF_S (Suffisante), F_Institu (Institutionelle), F_Tice_Oui, et d'autre part, le contraire de ces modalités (du côté des composantes positives), à savoir, E_Interact_Non , O_Tice_NF , E_Tice_Non, OF_INS (Insuffisante), F_Auto (Autoformation), F_Tice_Non. 


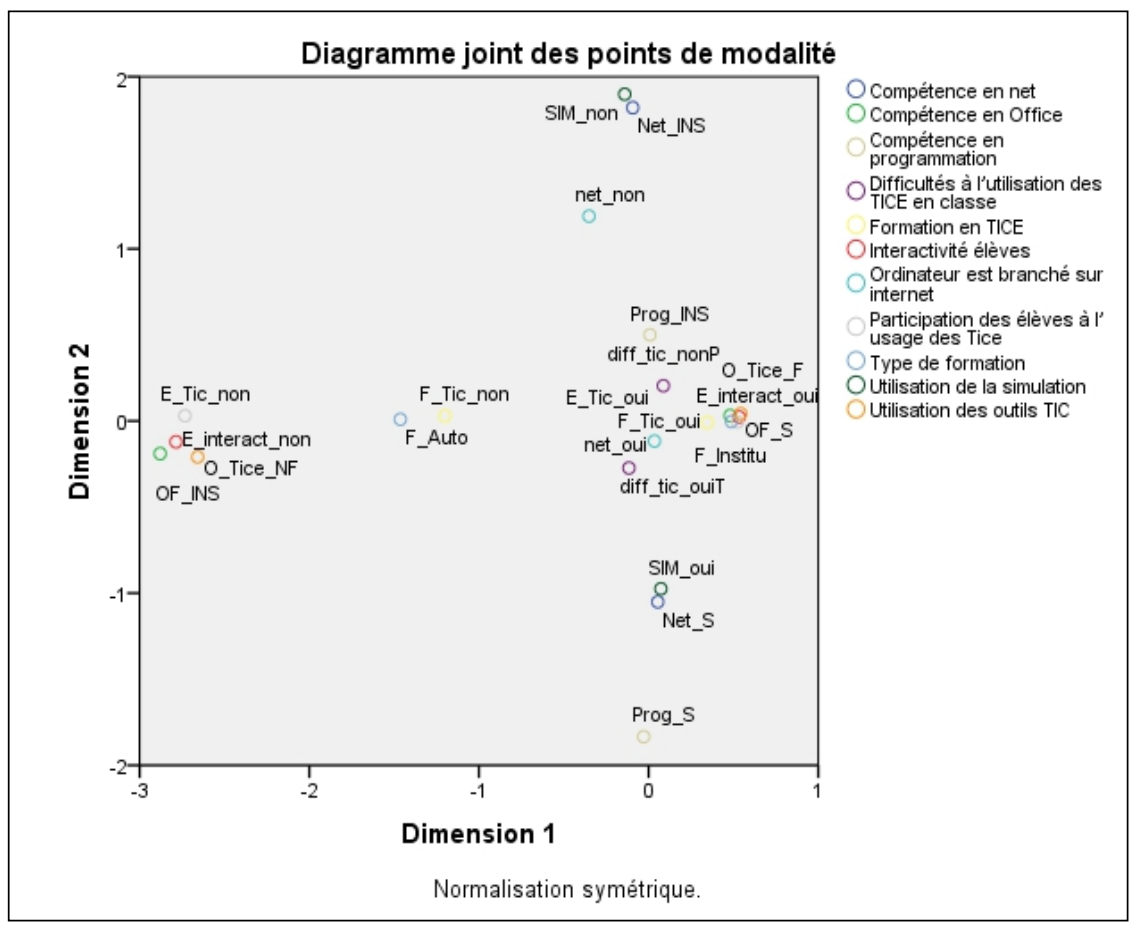

Figure 1 : Diagramme des modalités des variables : Premier plan factoriel (Axe1, Axe2)

Dans le premier plan factoriel (Axe1, Axe2) (Voir Figure 1), on constate qu'il y a une parfaite opposition par rapport au $1^{\mathrm{er}}$ axe factoriel entre les modalités représentant la présence (Oui) et l'absence (Non) de ces variables. Ainsi, le premier axe factoriel sépare, d'une part (du côté des composantes négatives) les modalités E_Interact_Oui , O_Tice_F , E_Tice_Oui, OF_S (Suffisante), F_Institu (Institutionelle), F_Tice_Oui, et d'autre part, le contraire de ces modalités (du côté des composantes positives), à savoir, E_Interact_Non , O_Tice_NF , E_Tice_Non, OF_INS (Insuffisante), F_Auto (Autoformation), F_Tice_Non.

Cette opposition parfaite entre ces deux groupes opposés de modalités permet dans un premier temps de conclure le fait que le questionnaire que nous avons utilisé pour explorer la question d'intégration des TIC dans l'enseignement, est bien construit et cohérent dans son ensemble au regard de ladite problématique.

Les variables correspondant aux plus fortes contributions relatives à la construction du $1^{\mathrm{er}}$ axe factoriel sont, selon leur ordre d'importance (Cf. Tableau 1) :
- $\quad$ Interactivité des élèves
- $\quad$ Utilisation des Outils TICE
- $\quad$ Participation des élèves à l'usage des TICE
- $\quad$ Compétence en Office 
L’interprétation du $1^{\mathrm{er}}$ axe factoriel devient ainsi immédiate, il s’agit de l'axe représentant " l'apport pédagogique et les conditions fondamentales d'une réussite de l'intégration des TICE ». En effet, si l'on tient compte des deux autres variables («Type de formation » et « Formation en TICE ») qui ont aussi contribué à la construction du $1^{\mathrm{er}}$ axe factoriel, on constate que cet axe traduit la réussite de l'intégration des TICE par :

- En premier lieu, son apport pédagogique en termes d' « Interactivité des élèves », à travers leur appropriation de l'environnement numérique qui s'exprime dans le premier axe factoriel par la « Participation des élèves à l'usage des TICE »,

- $\quad$ Ensuite, des conditions fondamentales à cette réussite, à savoir l'utilisation des outils TICE dans l'enseignement (modalité O_Tice_F de la variable «Utilisation des Outils TICE »), qui doit être prise en charge par l'enseignant. Celui-ci doit avoir de préférence une formation institutionnelle (modalités F_Tice_Oui et F_Institu des variables respectives « Formation en TICE » et « Type de formation »), notamment à propos du pack Office (modalité OF_S (Suffisante) de la variable «Compétence en Office »).

On peut donc déjà conclure d'après l’interprétation du premier axe factoriel, qu'une réussite de l'intégration des TICE dans un enseignement de SVT, et très probablement pour toute autre discipline scientifique, est avant tout tributaire d'un aspect purement didactique, à savoir « l'interactivité des élèves » dans un environnement numérique intégré par les élèves euxmêmes. La réussite d'une telle intégration nécessite des conditions minimales du côté de l'enseignant : une maîtrise des logiciels du pack Office (Word, Excell, PowerPoint, Paint, Photoshop,...), suite à une formation institutionnelle (par opposition à une autoformation) ; ce qui représente en effet un des volets importants du programme GENIE.

\section{Interprétation du $2^{\text {ème }}$ axe factoriel}

L’AFCM a conduit à une inertie absolue de 19,78\% pour le 2ème axe factoriel. Le tableau suivant résume les variables ayant les plus fortes contributions relatives au deuxième axe factoriel :

Tableau 2 : Variables à fortes contributions relatives dans la construction du $2{ }^{\text {ème }}$ axe factoriel

\begin{tabular}{|c|c|}
\hline Intitulé des variables (Modalités) & $\begin{array}{c}\text { Contribution relative } \\
\text { à l'Axe 2 }\end{array}$ \\
\hline Compétence en Net (Net_S (Suffisant) et Net_INS (Insuffisant)) & 0,850 \\
\hline Utilisation de la simulation (SIM_Oui_F et SIM_Non) & 0,823 \\
\hline $\begin{array}{c}\text { Compétence en programmation (Prog_S (Suffisant) et Prog_INS } \\
\text { (Insuffisant)) }\end{array}$ & 0,408 \\
\hline
\end{tabular}

Comme on peut le constater dans le premier plan factoriel (Voir Figure 1), il y a là encore une parfaite opposition entre, d'une part les modalités qui représentent une présence suffisante des compétences relatives 
aux trois variables « Compétence en Net », « Utilisation de la simulation » et « Compétence en programmation », avec des composantes positives sur le second axe factoriel, et d'autre part les modalités correspondantes qui représentent l'absence de compétence relatives aux dites trois variables, avec des composantes négatives par rapport au deuxième axe factoriel. Cette parfaite opposition, confirme encore une fois de plus, la bonne cohérence du questionnaire utilisé.

Le deuxième axe s'interprète naturellement comme étant l'axe des « Compétences avancées en TIC et Informatique ». En effet, la plus forte contribution relative à ce second axe renvoie à la variable «Compétence en Net », ce qui traduit bien entendu une compétence fondamentale dans la réussite de l'intégration des TICE. Quand aux deux autres variables qui ont contribué à la construction de cet axe, «Utilisation de la simulation » et « Compétence en programmation », elles font référence à des compétences avancées en informatique. Ainsi, le second axe factoriel, met en avant d'autres conditions supplémentaires en charge de l'enseignant, à savoir des compétences à la fois en TIC et en informatique : les compétences en Net, Simulation et programmation informatique, semblent se positionner au deuxième rang par rapport aux conditions fondamentales mises en avant par le premier axe factoriel, mais elles représentent des compétences avancées pour une meilleure intégration des TICE dans l'enseignement, notamment lorsqu'il s'agit des Sciences de la Vie et de la Terre (SVT), ou de toute autre enseignement relatif à une discipline expérimentale.

\section{Validation de l'interprétation du premier plan factoriel (Axe1, Axe2)}

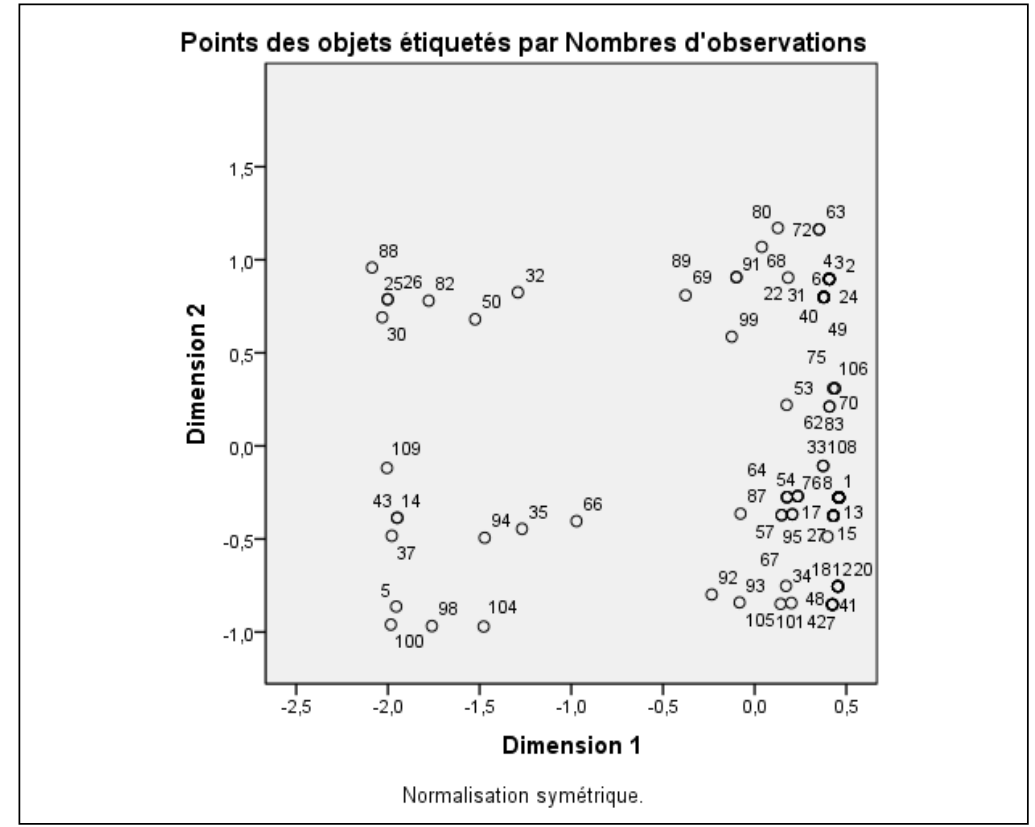

Figure 2 : Projection des individus sur le premier plan factoriel (Axe1 , Axe2) 
La projection des individus interrogés sur le premier plan factoriel confirme les interprétations données aux deux premiers axes factoriels 1 et 2 (Voir Figure 2). En effet, en allant chercher les individus qui possèdent les plus fortes contributions relatives au premier plan factoriel (Axe1 , Axe2), avec la meilleure qualité de représentation, nous avons obtenu les résultats suivants :

Tableau 3 : Individus à fortes contributions relatives dans la construction du premier plan factoriel

\begin{tabular}{|c|c|c|c|c|c|c|}
\hline Individus & $\begin{array}{c}\text { Contribution } \\
\text { /Axe1 }\end{array}$ & $\begin{array}{c}\text { Qualité } \\
\text { /Axe1 }\end{array}$ & $\begin{array}{c}\text { Contribution } \\
\text { / Axe2 }\end{array}$ & $\begin{array}{c}\text { Qualité } \\
\text { /Axe2 }\end{array}$ & $\begin{array}{c}\text { Composante } \\
\text { /Axe1 }\end{array}$ & $\begin{array}{c}\text { Composante } \\
\text { /Axe2 }\end{array}$ \\
\hline 30 & $\mathbf{0 , 0 5 9}$ & $\mathbf{0 , 8 5 8}$ & $(0,010)$ & $(0,070)$ & $\mathbf{2 , 5 6 3}$ & $\mathbf{- 1 , 0 3 5}$ \\
\hline 63 & $(0,02)$ & $(0,052)$ & $\mathbf{0 , 0 2 7}$ & $\mathbf{0 , 4 0 7}$ & $\mathbf{- 0 , 4 4 2}$ & $\mathbf{- 1 , 7 4 4}$ \\
\hline 72 & $(0,02)$ & $(0,052)$ & $\mathbf{0 , 0 2 7}$ & $\mathbf{0 , 4 0 7}$ & $\mathbf{- 0 , 4 4 2}$ & $\mathbf{- 1 , 7 4 4}$ \\
\hline
\end{tabular}

L’individu « 30 » est celui qui possède la plus forte contribution au premier plan factoriel, avec une contribution relative au premier axe de 0,059 (et une qualité de représentation / Axe1 de 0,858). Les deux composantes de cet individu dans le premier plan factoriel sont négatives et importantes en valeur absolue : l'analyse du profil des réponses de cet individu montre qu'il possède un ordinateur connecté à internet; en revanche, cet individu a répondu par « Non » à tout le reste des items du questionnaire : c'est le profil même de l'enseignant extrême qui rejette complètement l'intégration des TIC dans l'enseignement, et de surcroit toute formation relative au TICE, qu'elle soit d'ordre institutionnel ou personnel. Ce profil confirme totalement l’interprétation attribuée au premier axe factoriel.

Les deux individus « 63 » et « 72 » ont exactement la même contribution relative au premier plan factoriel, avec la plus forte contribution relative au deuxième axe factoriel, d'une valeur de 0,027, ainsi que la meilleure représentation sur cet axe factoriel $(0,407)$. Ces deux individus ont exactement le même profil : ils possèdent chacun un ordinateur qui n'est pas connecté à internet, et ont suivi chacun une formation institutionnelle en TICE. Ils possèdent des compétences en Pack Office, et intègrent ses outils dans leurs enseignements, durant lesquels les élèves interagissent, tout en s’appropriant les outils TIC utilisés. Néanmoins, ces deux enseignants n’ont aucune compétence ni en Net, ni en programmation (ce qui n’est pas étonnant puisqu'ils ne sont pas connectés à Internet !). Les composantes négatives des projections de ces deux individus dans le premier plan factoriel, confirment à leur tour l'interprétation donnée au second axe factoriel, en toute cohérence avec celle attribuée au premier axe factoriel.

\section{Interprétation du $3^{\text {ème }}$ axe factoriel}

L’AFCM a conduit à une inertie absolue de $9,87 \%$ pour le $3^{\text {ème }}$ axe factoriel. Les variables qui ont le plus contribué à la construction de cet axe sont les suivantes : 
Tableau 4 : Variables à fortes contributions relatives dans la construction du $3^{\text {ème }}$ axe factoriel

\begin{tabular}{|c|c|}
\hline Intitulé des variables (Modalités) & $\begin{array}{c}\text { Contribution } \\
\text { relative à l'Axe 3 }\end{array}$ \\
\hline Ordinateur branché sur Internet (Net_Oui et Net_Non) & 0,379 \\
\hline Formation en TICE (F_Tice_Oui et F_Tice_Non) & 0,265 \\
\hline $\begin{array}{c}\text { Difficulté à l'utilisation des TICE en classe (Diffi_TIC_Non et } \\
\text { Diffi_TIC_Oui) }\end{array}$ & 0,261 \\
\hline
\end{tabular}

Le troisième axe factoriel oppose les modalités Net_Oui, F_Tice_Oui et Diffi_TIC_Oui (toutes à composantes positives sur l'Axe3), face aux modalités Net_Non, F_Tice_Non et Diffi_Tic_Oui (toutes à composantes négatives sur l'Axe3).

Cette opposition nous informe sur la «Difficulté d'utilisation des TICE en classe ». En effet, le regroupement des modalités Net_Non, F_Tice_Non et Diffic_TIC_Non, s'interprète très facilement : un enseignant non connecté à Internet, et qui par ailleurs n'a pas suivi de formation sur les TICE, n'aura pas tendance à intégrer les TICE en clase, d'où l'absence de difficulté à l'utilisation des TICE en classe. En revanche, le regroupement des modalités Net_Oui, F_Tice_Oui et Diffi_TIC_Oui, nous informe sur une difficulté à utiliser les TICE en classe : Bien que les enseignants aient suivi une formation sur les TICE, cela ne suffit pas apparemment pour surmonter les difficultés de gestion de classe: la formation en TICE devrait donc obligatoirement être accompagnée de formations autour de la pédagogie et de la didactique d'un enseignement intégrant les TIC.

\section{Conclusion et perspectives de la recherche}

L'approche méthodologique retenue dans notre recherche pour l'étude de l'intégration des TICE en Sciences de la vie et de la Terre (SVT) au secondaire qualifiant, diffère totalement de celles des études antérieures. En effet, celles-ci sont toutes basées sur de la description statistique, avec des statistiques unidimensionnelles, ou de l'application de tests statistiques d'indépendance pour des statistiques bidimensionnelles. Notre approche méthodologique est en revanche basée sur la multidimensionnalité, avec pour objectif l'identification et la quantification de toutes les liaisons importantes issues de l'ensemble des items de notre questionnaire.

L'analyse factorielle de Correspondance Multiple (AFCM) appliquée aux données recueillies par l'interrogation de 112 enseignants a permis effectivement de construire trois axes factoriels qui cumulent $70 \%$ de la part d'information contenue dans l'ensemble des réponses des enseignants interrogés. La hiérarchie induite par les contributions absolues a donc permis de donner à chacun de ces axes, selon leur ordre d'importance, les interprétations suivantes : 
- Le premier axe factoriel, avec une contribution absolue de $40 \%$, représente "l'apport pédagogique et les conditions fondamentales d'une réussite de l'intégration des TICE »

- Le second axe factoriel, avec une contribution absolue de 20\%, représente "les compétences avancées en TIC et Informatique »

- Le troisième axe factoriel, avec une contribution absolue de $10 \%$, représente "Les difficultés d'utilisation des TICE en classe "

Contre toute attente, nous constatons que l'interactivité des élèves en classe, est l'item qui a la plus forte contribution relative dans la construction du premier axe factoriel. Ce résultat souligne la grande importance de l'appropriation des élèves de leur environnement numérique ; cela représente une condition fondamentale pour la réussite de l'intégration des TICE. Une formation en TICE, de préférence institutionnelle (par opposition à une autoformation), ainsi qu'une connaissance du Pack Office, sembleraient être aussi des conditions fondamentales minimales pour une bonne intégration des TICE. Ainsi, l'analyse multidimensionnelle a fait apparaître, à un titre au moins de même importance que la formation des enseignants en TICE, le fait d'initier et de faire adhérer les élèves aux outils TIC. Cela suppose donc qu'il devient important aujourd'hui d'intégrer dans les curricula des élèves des enseignements faisant appel au TIC, comme par exemple l'algorithmique ou encore des applications pouvant permettre de réaliser des simulations de modèles théoriques. De tels enseignements participeront certainement à une bonne appropriation de la part des élèves des environnements numériques, ainsi qu'à leur bonne adaptation aux pédagogies intégrant les TIC en classe.

Des compétences avancées en TIC et informatique semblent aussi avoir un effet très positif sur l'intégration des TICE. Ainsi, les items qui ont le plus contribué à la mise en avant de ce facteur sont essentiellement des compétences en Net et en programmation, ainsi que l'utilisation de la simulation en classe. A ce titre là, le programme GENIE répond parfaitement à ces attentes par le biais de diverses formations institutionnelles ; c'est donc une des actions politiques menées par le Ministère de l'Education qui est en parfait accord avec une bonne réussite de l'intégration des TICE.

Enfin, un dernier élément fondamental à l'intégration des TICE, et qui a été mis en avant par le troisième axe factoriel, concerne la formation des enseignants sur les pédagogies et didactiques d'intégration des TICE en classe. En effet, une formation sur les outils TIC, qu'elle soit institutionnelle ou personnelle, ne semble pas suffire pour surmonter les difficultés de gestion de classe; les nouveaux aspects pédagogiques et didactiques de gestion de classe induits par l'intégration des TIC, doivent eux aussi être pris en charge lors des formations institutionnelles.

Nous pensons que la majorité des résultats de cette recherche pourra s'appliquer à plusieurs autres disciplines scientifiques. Une des perspectives 
de cette recherche serait de conduire le même type d'analyse auprès d'enseignants de mathématiques et de sciences physiques. La comparaison de telles recherches permettra d'identifier un certain nombre de variables invariantes relatives à la problématique de l’intégration des TICE au Maroc. Une telle validation de variables permettra alors d'accomplir une grande avancée dans le traitement de la question de l'intégration des TICE dans l'enseignement secondaire marocain.

\section{References:}

Agence Française pour le Développement - AFD (2010) : Bilan critique en matière d'utilisation pédagogique des NTIC dans le secteur de l'éducation. Rapport final.

Alj, O. et Benjelloun, N. (2016) : Etude comparative de quelques recherches sur l'intégration des TIC dans l'enseignement des disciplines scientifiques au sein du programme GENIE marocain. Association EPI. epi.asso.fr/revue/articles/a1601c.htm

Alj, O. (2014) : Etude de l'intégration des TIC dans le cadre du programme GENIE marocain : attitudes des enseignants et impact dans les pratiques d'enseignement scientifique. Thèse de Doctorat, Laboratoire Interdisciplinaire de Recherche en Didactique des Sciences et Techniques (LIRDIST), Faculté des Sciences Dhar El Mahraz, Université Sidi Mohammed Ben Abdellah, Fès.

COSEF Maroc, (1999) : La Charte nationale de l'éducation et de formation.

Droui, M. (2015) : Simulation et expérimentation assistée par ordinateur pour l'enseignement de la physique: élaboration, expérimentation et évaluation des situations d'apprentissages. Thèse de Doctorat, Laboratoire Interdisciplinaire de Recherche en Didactique des Sciences et Techniques (LIRDIST), Faculté des Sciences Dhar El Mahraz, Université Sidi Mohammed Ben Abdellah, Fès.

El Ouidadi, O. (2012) : Etat des lieux et enjeux de l’implémentation des TICE dans le système scolaire marocain : Profils des enseignants et étude d'impact en SVT. Thèse de Doctorat, LADIPEC, Faculté des Sciences Dhar El Mahraz, Université Sidi Mohammed Ben Abdellah, Fès.

El Madhi, Y. ; Chiahou, B. ; Belghyti, D. ; El Kharrim, K. ; El Halouani H.(2014) : Les contraintes liées a l'integration du tic dans l'enseignement des sciences de la vie et de la terre au maroc. European Scientific Journal edition vol.10, No.34 ISSN: 1857 - 7881.

Eurydice (2011): L'enseignement des sciences en Europe: politiques nationales, pratiques et recherche. Education, Audiovisual and Culture Executive Agency.

Lakdim, A. ; El Ouidadi, O. ; Essafi, K. et Sendide, K. (2012) : Etude de l'impact de l'utilisation d'une plate-forme en ligne pour la régulation des 
apprentissages et l'accompagnement des élèves en SVT : Cas des lycées de la ville de Fès (Maroc). http://www.epi.asso.fr/revue/articles/a1204b.htm Ouazzani Touhami, A. (2014) : Etude des difficultés concveptuelles relatives à la construction d'une image virtuelle et impact des TICE sur les productions des apprenants. Thèse de Doctorat, Laboratoire Interdisciplinaire de Recherche en Didactique des Sciences et Techniques (LIRDIST), Faculté des Sciences Dhar El Mahraz, Université Sidi Mohammed Ben Abdellah, Fès.

Ouazzani Touhami, A., Benjelloun, N., Aami, M. and Haddou, A. (2014) : Impact of using Java Software of Geometrical Optics JSGO on the construction success of the virtual image among the first year university students. IOSR Journal of Research \& Method in Education (IOSR-JRME), Volume 4, Issue 3 Ver II, pp. 16-24.

Maouni, A. ; mimet, M. ; khaddor, M. ; madrane, M. ; moumene. (2014) : L'intégration des TIC dans l'enseignement des SVT au Maroc : réalité et attentes. RADISMA, Numéro 10.

Matoussi, F. (2006): Les technologies de l'information et de la communication intégrées dans l'enseignement de la biologie. Le cas des échanges cellulaires. Thèse pour l'obtention de diplôme de docteur en didactique ; Université de Toulouse II Le-Mirail. 
Annexe A

Questionnaire

I.Usage personnel des TICE

Q 1 : Avez-vous un ordinateur à domicile?

Q 2 : Si oui, est ce que cet ordinateur est branché sur Internet ?

Q 3 : Quelle est votre fréquence quotidienne de navigation sur Internet?

$\square$ Pas tous les jours

Moins d'une heure par jour

$\square$ Plus que deux heures par jour

II.Disponibilité et utilisation des TIC dans l'enseignement

Q 4 : Avez-vous une salle informatique dans votre établissement?

Q 5 : Quel est votre degré d'utilisation des outils pédagogiques suivants?

\begin{tabular}{|c|c|c|}
\hline & Fréquemment & Non Fréquemment \\
\hline Rétroprojecteur & & \\
\hline Audio-Vidéo & & \\
\hline EXAO & & \\
\hline Internet & & \\
\hline Tableau blanc interactif mobile & & \\
\hline
\end{tabular}

Q 6 : Utilisez-vous de la simulation dans vos cours?

Q 7 : Quel est le cours ou l'activité en SVT où vous souhaiteriez utiliser de la simulation ?

Q8 : Utilisez-vous des outils TIC pour la planification de vos cours?

Jamais occasionnellement régulièrement

Toujours

\section{Gestion pédagogique des TIC en classe}

Q9 : Lors de votre utilisation des outils TIC en classe, est ce que vous amenez vos élèves à faire usage aussi des TIC ?

Q10 : Comment les élèves interagissent-ils avec un cours utilisant les TIC ?

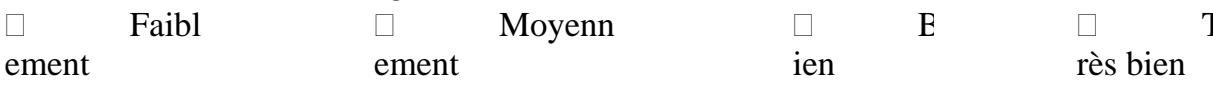

Q11 : Quelles sont les difficultés rencontrées lors de l'utilisation des TIC dans la classe ?

IV. Formations et compétences en TICE et Informatique

Q 12 : Avez-vous suivi ou bénéficié d'une formation sur l'utilisation des TICE ?

Q 4 : Si oui, quel est le type de cette formation?

Institutionnel

Autonome

Q14 : Quel est votre degré de maîtrise du Pack « Office » :

Suffisante Insuffisante

Q15 : Quel est votre degré de maîtrise d' « internet » :
Suffisante
Insuffisante

Q16 : Quel est votre degré de maîtrise en «programmation» :

Suffisante

Insuffisante 


\section{Annexe B}

\section{Résultats de l’AFCM (avec 11 modalités)}

Récapitulatif de traitement des observations

Observations valides actives
Observations actives avec valeurs manquantes

Observations supplémentaires

Total

Observations utilisées dans l'analyse

\begin{tabular}{|c|}
\hline 112 \\
0 \\
0 \\
112 \\
112 \\
\hline
\end{tabular}

Historique des itérations

\begin{tabular}{|c|c|c|c|}
\hline \multirow{2}{*}{ Numéro de l'itération } & \multicolumn{2}{|c|}{ Variance expliquée } & \multirow{2}{*}{ Perte } \\
\cline { 2 - 3 } & Total & Augmentation & \\
\hline $66^{\mathrm{a}}$ & 2,532992 &, 000010 & 8,467008 \\
\hline
\end{tabular}

a. Le processus d'itération s'est interrompu car la valeur test de la convergence a été atteinte.

Récapitulatif des modèles

\begin{tabular}{|c|c|c|c|c|}
\hline \multirow{2}{*}{ Dimension } & Alpha de & \multicolumn{3}{|c|}{ Variance expliquée } \\
\cline { 3 - 5 } & Cronbach & $\begin{array}{c}\text { Total (valeur } \\
\text { propre) }\end{array}$ & Inertie & $\begin{array}{c}\text { Pourcentage de variance } \\
\text { expliquée }\end{array}$ \\
\hline 1 &, 846 & 4,338 &, 394 & 39,433 \\
2 &, 595 & 2,176 &, 198 & 19,783 \\
3 &, 086 & 1,085 &, 099 & 9,865 \\
Total & & 7,599 &, 691 & 23,027 \\
Moyenne &, $666^{\text {a }}$ & 2,533 &, 230 & 2 \\
\hline
\end{tabular}

a. La valeur Alpha de Cronbach moyenne est basée sur la valeur propre moyenne.

Mesures de discrimination

\begin{tabular}{|c|c|c|c|c|}
\hline & \multicolumn{3}{|c|}{ Dimension } & \multirow[t]{2}{*}{ Moyenne } \\
\hline & 1 & 2 & 3 & \\
\hline $\begin{array}{l}\text { Ordinateur est branché sur } \\
\text { internet }\end{array}$ & 008 & ,062 & ,379 & , 149 \\
\hline $\begin{array}{l}\text { Difficultés à l'utilisation } \\
\text { des TICE en classe }\end{array}$ & ,006 & ,025 & 261 & ,098 \\
\hline Utilisation des outils TIC & 907 & ,004 & ,009 & ,307 \\
\hline $\begin{array}{c}\text { Participation des élèves à } \\
\text { l'usage des Tice }\end{array}$ & 898 & ,000 & ,019 & ,306 \\
\hline Interactivité élèves & 933 & 001 & ,015 & 317 \\
\hline Formation en TICE & ,260 & ,000 & ,265 & ,175 \\
\hline Type de formation & 448 & ,000 & ,066 & 171 \\
\hline Compétence en Office & 867 & ,003 & ,007 & 292 \\
\hline Compétence en net & ,003 & ,850 & ,022 & ,292 \\
\hline $\begin{array}{l}\text { Compétence en } \\
\text { programmation }\end{array}$ & 000 & ,408 & ,007 & 138 \\
\hline $\begin{array}{l}\text { Utilisation de la } \\
\text { simulation }\end{array}$ & ,006 & 823 & ,034 & ,288 \\
\hline Total actif & 4,338 & 2,176 & 1,085 & 2,533 \\
\hline $\begin{array}{c}\text { Pourcentage de variance } \\
\text { expliquée }\end{array}$ & 39,433 & 19,783 & 9,865 & 23,027 \\
\hline
\end{tabular}

